\title{
THEORETICAL STUDIES IN NUCLEAR STRUCTURE
}

\author{
Progress Report \\ for Period June 1, 1991 - November 30, 1991
}

E. R. Marshalek

University of Notre Dame

Notre Dame, IN 46556

\section{DISCLAIMER}

\begin{abstract}
This report was prepared as an account of work sponsored by an agency of the United States Government. Neither the United States Government nor any agency thereof, nor any of their employees, makes any warranty, express or implied, or assumes any legal liability or responsibility for the accuracy, completeness, or usefulness of any information, apparatus, product, or process disclosed, or represents that its use would not infringe privately owned rights. Reference herein to any specific commercial product, process, or service by trade name, trademark, manufacturer, or otherwise does not necessarily constitute or imply its endorsement, recommendation, or favoring by the United States Government or any agency thereof. The views and opinions of authors expressed herein do not necessarily state or reflect those of the United States Government or any agency thereof.
\end{abstract}

\section{November 1991}

Prepared for 


\section{NOTICE}

This report was prepared as an account of work sponsored by the United States Government. Neither the United States nor the Department of Energy, nor any of their employees, nor any of their contractors, subcontractors, or their employees, makes any warranty, express or implied, or assumes any legal liability or responsibility for the accuracy, completeness, or usefulness of any information, apparatus, product or process disclosed, or represents that its use would not infringe privatelyowned rights. 


\begin{abstract}
In this period, the work has centered on two topics. The first is the study of a novel type of collective rotation in which an atomic nucleus with an inversion-symmetric shape rotates uniformly about an axis that is not a principal axis of the quadrupole tensor of the density distribution. This mode is referred to as tilted rotation. By using the cranking model together with higher-order corrections, it was shown that tilted rotation is indeed possible, not only within a microscopic framework, but also within the framework of collective models such as the IBM. The maximum tilt angle of $\pi / 4$ is realized for a certain class of states in the $U(5)$ limit. The second topic, which actually was suggested during the course of the first investigation, is concerned with a new way of representing collective harmonic-oscillator algebras using boson-mapping techniques. In this approach, the many-phonon eigenvectors of a $2 \lambda+1$-dimensional oscillator having good angular momentum are represented by simple products of boson operators acting on a vacuum. This representation may simplify the calculation of reduced matrix elements of arbitrary operators in col'ective models, but more work needs to be done.
\end{abstract}

\title{
A. TILTED ROTATION
}

Collective rotational motion has been known in atomic nuclei for over forty years. Studies of this phenomenon have been greatly invigorated by the recent discovery of rotational bands in superdeformed nuclei extending to very high angular momenta. ${ }^{1}$ The premier theoretical approach used to elucidate this phenomenon is the semiclassical cranking model, which usually assumes that the nucleus rotates uniformly about the principal axis of the mass quadrupole tensor corresponding to the largest moment of inertia. For a rigid body, rotation about a principal axis is the only mode of uniform rotation possible. But a nucleus is not a rigid body. In that case, other possibilities may arise. As discussed in Chandrasekhar's classic treatise on ellipsoidal equilibrium shapes of rotating gravitational fluids, ${ }^{2}$ the Riemann type-II family corresponds to uniform rotation about an axis that lies in a principal plane but does not coincide with a principal axis. In other words, the rotation occurs abrut an axis that is tilted with respect to a principal axes. As it turns out, this family of equilibrium solutions is unstable, based on a small-oscillation criterion. The question arises whether examples of titled rotation might occur in nuclei, and, in the case they should also turn out to be unstable, whether the instability necessarily implies that the mode is unphysical. 
The possibility of rotation about a tilted axis within the framework of the particle-rotor model was alluded to by Hamamoto in $1984 .^{3}$ Three years later, tilted solutions were found for odd-A and odd-odd nuclei by Bengtsson and Frisk, ${ }^{4}$ using the cranked shell-model. Although the calculations were very limited in scope, they indicated that tilted rotation is a very definite possibility owing to the asymmetry introduced by odd nucleons. For example, in the odd-odd case, the orbital of one nucleon may energetically favor rotation about one principal axis while the orbital of the other nucleon favors another axis, so that the tilted axis emerges as a compromise. Unlike the Riemann solution, these solutions are yrast bands for some range of angular momenta. Although the stability has not been checked, it seems likely that the Bengtsson-Frisk solutions are true minima, since otherwise they probably could not be located by the technique used. This line of work has recently been taken up anew by Frauendorf. 5

There is still the question of whether tilted rotation can occur for even-even nuclei. Now, I had found an example of this over twenty years ago while studying the self-consistent cranked mean-field method in spherical nuclei. Spherical nuclei, i.e., nuclei that are spherical in the ground state, may seem unlikely candidates for the cranking approach. However, if cranlecd with a sufficiently large angular speed, deformed solutions do arise. ${ }^{6}$ Thus, in spherical nuclei, unlike permanently deformed ones, there is a nonzero threshhold angular frequency. In this case, I found that the cranking method portrays certain subfamilies of multiphonon states as rotational bands. One family, which is often an yrast band and will be referred to hereafter as the "Y band", has a spin sequence given by $I=2 n_{\mathrm{d}}$, where $n_{\mathrm{d}}$ is the number of quadrupole or $d$ phonons, and corresponds to what is often called a "quasirotational band". This type of solution corresponds to a normal rotation about a principal axis. However, other families correspond to tilted rotation. The work on the tilted solutions was not published at the time since it was not clear whether the solutions were physical and, in any case, the chances of detecting such bands was probably beyond the experimental technology of that period. 
My interest in the subject was rekindled not only by the work cited in Refs. 3 and 4 but also by a recent paper of Cuypers $^{7}$, who used the self-consistent cranking model to derive extremum equations describing uniform rotation of nucleons moving in the volume-conserving oscillator potential. These equations allow for both normal and tilted rotation. Unfortunately, the author, having found the former solutions, in a lapse of logic toward the end of the paper concluded that they were the only solutions, i.e., that tilted rotation does not exist in the oscillator shell model. However, using a perturbation technique and computer algebra (REDUCE), I found that tilted solutions indeed do exist in this model for closed-shell configurations and that these correspond exactly to the class of solutions I had found earlier for spherical nuclei in a more general context. These solutions, like those of Riemann, have the rotation axis in a principal plane. The tilt angle is quite pronounced, approaching $45^{\circ}$. The possibility of tilted rotation for other configurations remains to be investigated. Although the tilted rotation is indeed an equilibrium solution, the inability of numerical energy-minimization codes to ixcate it suggested that it might be unstable. While the stability problem in microscopic models can (and will) be investigated more rigorously by small-oscillation theory (RPA), it seemed more useful at this early stage to investigate the corresponding modes in simpler exactly soluble models, namely phenomenological collective models.

It is quite straightforward to apply cranking techniques to collective models. Indeed, I was able to find the analogs of the above solution for both the Copenhagen-Frankfurt model and the interacting boson model (IBM) ${ }^{8}$ near the spherical or U(5) limit. In the U(5) limit the tilt angle approaches $45^{\circ}$. The tilted solution, referred to hereafter as the "T band", describes the band of states for which the spin $I=n_{\mathrm{d}}$, and $n_{\beta}=0$, where $n_{\mathrm{d}}$ is the number of $d$ bosons and $n_{\beta}$ is the $\mathrm{O}(5)$ quantum number defined in Ref. 8. It is important to note that although the density distribution is tilted (based on the conventional geometric interpretation of the IBM), the inertia tensor adjusts so that it becomes isotropic about the axis of rotation. It is precisely this indifference of the inertia tensor that enables the tilting to occur, a mechanism that is absent in a rigid rotator. There is also a normal (quasi-) rotational or $\mathrm{Y}$ band for which $I=2 n_{\mathrm{d}}, n_{\beta}=0$. The accuracy of the cranking method for both solutions is just what one 
would expect for a basically classical approximation, with errors of the order of $I^{-1}$.

In order to assess the stability question, small oscillations about the cranked solutions were studied specifically for the IBM, which is equivalent to doing the RPA. It was found that the $\mathrm{T}$ band solution, like the Riemann solution, is indeed unstable for any choice of parameters, as manifested by an RPA Hamiltonian that is not a positive quadratic form. This is due to the presence in this Hamiltonian of a normal mode of the form $\hbar \omega C^{\dagger} C$ with negative frequency $\omega$. Here, $C^{\dagger}$ is a boson operator representing an excitation built on the $Y$ band as the reference state. However, since this band is never yrast, the negative-frequency mode simply allows for de-excitation from the tilted band to bands below, including the yrast one. The conciusion then is that while the negative-frequency mode formally signifies a kind of instability, there is nothing unphysical about it. Of course, one must be careful not to apply $C^{\dagger}$ too many times to avoid going below the yrast line. This is very different from having an imaginary frequency, which would indeed signify an unphysical solution.

In order to check this idea further, the analysis was carried out one order beyond the RPA. The problem of broken symmetry generated by the deformed cranking solution and the attendant Goldstone modes was handled by the Marshalek-Weneser (MW) method. ${ }^{9}$ The result, as compared to the exact IBM solutions obtained from group theory, is complete agreement for all eigenvalues. This also provides a nice confirmation for the MW prescription as well. An account of this work was recently presented at an invited talk at the Symposium on Contemporary Physics held at Drexel University, Philadelphia (Oct. 31 - Nov. 1, 1991) in honor of the $65^{\text {th }}$ birthday of Professor Abraham Klein. A manuscript of this talk will 'be prepared soon for publication in the proceedings of the Symposium. Much work remains to be done as discussed in Sec. C below.

\section{B. A NEW REPRESENTATION FOR COLLECITE OSCILLATORS}

Collective-model Hamiltonians and associated transition operators for even-even nuclei are constructed from boson operators. This is true not only of purely phenomenological 
models such as the Copenhagen-Frankfurt and IBM models, but also of microscopic shell models treated by boson-mapping techniques. ${ }^{10}$ In applications, the problem arises of calculating the matrix elements of the Hamiltonian and transition operators in a many-phonon basis. Since the basis vectors are required to be eigenstates of angular momentum, they are in general not simple products of boson operators acting on the vacuum, but have a much more complicated structure. Of course, techniques have been developed to solve this problem based on the use of fractional parentage coefficients together with group theory. In the case of quadrupole oscillators, i.e., $d$ bosons, certain matrix elements can even be obtained analytically with the aid of group theory, while others require extensive numerical computer evaluation. The latter point was driven home recently when I asked the Frankfurt group for help in adapting their collective-model Fortran program for high angular-momentum states and received a file of matrix elements that took two hours to download via modem. While this situation is not intolerable, it would be better if all matrix elements could be obtained analytically. For example, since the matrix elements depend on the total angular momentum $I$, one might be able to find new approximations for high-spin states.

As a spin-off (no pun intended) from the work described in the previous section, it was found that a new representation exists for the isotropic $2 \lambda+1$-dimensional oscillator in which the basis vectors are simple products of boson operators acting on the vacuum and eigenvectors of the total angular momentum at the same time. These boson operators are not the original ones, but a new set related to them by what is essentially a canonical transformation. The MW method mentioned previously provides such a transformation, but only as a power series in $I^{-1}$. This already provides the possibility of calculating arbitrary matrix elements for high-spin states to some order in $\Gamma^{-1}$, but the real challenge lies in summing the series. My idea is to use the boson-mapping techniques of Ref. 10 to map the original bosons (say the $d$ bosons for $\lambda=2$ ) into the new bosons corresponding to the simple product basis of angular-momentum eigenvectors.

As a pilot project, this program was carried through completely for the case $\lambda=1$, i.e., the three-dimensional oscillator, when one is dealing with $p$ bosons. Of course, in this case all 
matrix elements can be obtained analytically by other means as well. But that is all to the better, since it is possible then to verify the validity of the general approach. Also, the present method may be preferable to other techniques, such as the traditional use of tedious generating functions to calculate matrix elements for the three-dimensional oscillator, although that is a matter of taste.

For the case $\lambda=1$, one starts with the spectrum-generating algebra $\mathrm{SU}(1,1) \times \mathrm{SO}(3)$, where the two groups are generated by certain bilinear combinations of $p$ bosons. More specifically, $S U(1,1)$ corresponds to the boson seniority algebra ${ }^{11}$, while $S O(3)$ is just the rotation group. The generators of these two groups can be mapped into Dyson images involving the new bosons in a way that is readily generalizable to any $\lambda$. The corresponding Dyson images of single boson creation and annihilation operators can be obtained from the conditions that they are generators of the Heisenberg group and also tensors under $\mathrm{SU}(1,1) \times \mathrm{SO}(3)$. A surprising result is that these Dyson images are not unique, although their matrix elements are. As is well-known, a Dyson map is a nonunitary realization of a Lie algebra. It can be unitarized by standard techniques, which leads to what is called a (generalized) Holstein-Primakoff (HP) map. It turns out that the HP maps are unique, in spite of the nonuniqueness of the Dyson maps. The matrix elements of single boson creation and annihilation operators in the basis of good angular momentum may be read off directly from their HP maps. From these, the corresponding matrix elements of other operators can be analytically constructed. A manuscript of this work was submitted to the Journal of Mathematical Physics in September and a referee report is still being awaited.

The problem of extending this approach to quadrupole bosons is currently under investigation. A key step is the construction of the Dyson images of the $\mathrm{SU}(1,1)$ generators. This can be done for any $\lambda$ with the help of the following interesting observation made during the investigation discussed in Sec. A. If the cranking plus RPA technique is applied to an isotropic oscillator, one finds one or more partitions of the total number of quanta involving the angular-momentum quantum number. For example, for $\lambda=1$, the total number of quanta $n$ emerges in the form $n=I+2 n_{r}$, where $n_{r}$ is the eigenvalue of a normal mode number 
operator, which can be interpreted as the radial quantum number. For the case $\lambda=2$, one finds two distinct decompositions of $n\left(=n_{\mathrm{d}}\right)$, depending on whether the $\mathrm{Y}$ band or the $\mathrm{T}$ band is taken as the reference state. Corresponding to the T-band solution, one finds the decomposition $n=3 \tilde{n}_{\Delta}+2 n_{\beta}+I-n_{C}$, where the integers $\tilde{n}_{\Delta}, n_{\beta}$, and $n_{C}$ are eigenvalues of normal-mode boson number operators, the first two being interpreted in terms of familiar $U(5)$ quantum numbers, and one may take $I-n_{\mathrm{C}}=\lambda$, where $\lambda$ is the $\mathrm{U}(5)$ quantum number defined in Ref. 8 (and not the $\lambda$ used elsewhere in this report). Corresponding to the Y-band solution, one finds the alternative decomposition $n=2 n_{1}+n_{2}+3 n_{3} / 2+I / 2$, where the $n_{i}$ are integers, a partition known to some specialists in group theory. Given a decomposition of $n$, one can immediately construct a boson mapping of the $S U(1,1)$ generator $S_{0}$ (which is proportional to the oscillator Hamiltonian), and then use the algebra to obtain the Dyson or HP map of the remaining generators $S_{+}$and $S_{-}$. Corresponding to the T or $\mathrm{Y}$ bands then, one obtains two distinct but equivalent maps. In principle, one could then go on to obtain the corresponding maps of the Heisenberg generators as for the three-dimensional oscillator. However, while the general technique seems applicable, there are nontrivial hurdles, not only due to the greater complexity of the matrix elements, the calculation of which may require the use of computer algebra, but also with mysteries surrounding the so-called "missing label", denoted by $\tilde{n}_{\Delta}$ in Ref. 8.

\section{PROPOSED RESEARCH FOR THE BUDGET PERIOD}

The work on tilted rotation is still in the nascent stage but the interest in the subject is growing and much remains to be investigated. For example, just as this report was being prepared I learned that W. Nazarewicz at Oak Ridge Laboratory has also been studying tilted rotation, although he calls it "three-dimensional cranking". One of the most important questions, of course, is to find a unique tell-tale experimentally accessible characteristic to differentiate tilted from normal rotation. Since the tilted rotor no longer has the $180^{\circ}$ symmetry about the rotation axis, the signature quantum number is not a good quantum 
number for such a band as pointed out already in Ref. 4. That implies that all possible spins must be present in the band, unlike the spin sequence $I=0,2,4, \ldots$ in the ground band. However, that does not differentiate it from other well-known excited bands. Another possibility that deserves serious study is that of tunneling, which is suggested by the fact that for each tilted rotational configuration there is a mirror image. There may also be characteristic patterns in electromagnetic transitions, more so for $B(\mathrm{M} 1)$ 's than say $B(\mathrm{E} 2)$ 's. Unfortunately, the IBM-1 is not ideal for studying $B(\mathrm{M} 1)$ 's because the operator is, in the lowest approximation, proportional to the angular momentum in this model and therefore does not produce any transitions. Thus, it may be necessary to study the IBM-2 or other more complex versions in this regard.

Another important question is whether the IBM allows for tilted rotation at the other vertices of the Casten triangle, ${ }^{12}$ or, for that matter, in the interior of the triangle, and how the tilt angle (or angles, if the rotation axis should not lie in a principal plane) vary as one moves around the triangle. Is the same technique used at the $U(5)$ vertex - the cranked mean-field zeroth approximation followed by the RPA and higher order co.rections generated by the MW method - applicable at other points of the Casten triangle? There is yet another motivation $\mathrm{t}$ study this question, which is discussed in the next paragraph.

The IBM Hamiltonian is integrable at the vertices of the Casten triangle because it has a dynamical symmetry at those points. Inside the triangle, however, the dynamical symmetries disappear and the system becomes chaotic. Recently, Mizusaki, Yoshinaga and Shigahara ${ }^{13}$, using a two-parameter variant of the IBM, constructed a quantum chaos map of the Casten triangle based on energy-level statistics, which can always be obtained with the help of exact diagonalizations of the Hamiltonian. It would be very interesting to test the numerical accuracy of the MW method over the Casten triangle and its possible correlation with the chaos map. One of the reasons for this interest is that the MW method can be considered as a quantal analog of the Birkhoff-Gustavson method ${ }^{14}$, with the additional advantage of describing spontaneosly broken symmetries. The Birkhoff-Gustavson method is an approximation in which a nonintegrable classical Hamiltonian is expressed perturbatively as a 
power series in an oscillator Hamiltonian, which is suitable for an approximate quantization of the system. It can g،ve a good asymptotic series approximation to energy levels in regions where there are still invariant tori present, although its status in strongly chaotic regions is in doubt. The MW method gives a similar expansion in boson number operators and in constants of motion corresponding to broken symmetries. In contrast to the Birkhoff-Gustavson method, it is believed that the MW method includes all quantal corrections to a given order. For these reasons, it would be interesting to test the MW method throughout the Casten triangle. Such a test would also permit a better evaluation of its accuracy in realistic applications to microscopic systems.

If the phenomenological studies of tilted rotation suggest interesting experimental consequences, then certainly the next step would be the development of numerical codes for microscopic calculations, beginning with cranked mean fields and, hopefully expanding at least to include the RPA corrections.

While the study of tilted rotation will be the top priority for the next two months or so, the work on the quadrupole oscillator discussed above will also be continued. In addition, a renewed effort will be initiated to study the Coriolis attenuation in deformed odd-A nuclei, as outlined in my original proposal. There seems to be a rejuvenated interest in this old, important and unsolved problem. It may be interesting to calculate, not only in traditional deformed nuclei, but also in the superdeformed ones as well.

The time devoted to the project has been $100 \%$ in the summer and approximately $60 \%$ during the academic semesters. I also expect to spend an additional $15 \%$ of my time in the Spring semester to master the intricacies of the Unix operating system and my new workstation, but that, of course, will eventually pay off (one hopes) in greater computational productivity. 


\section{REFERENCES}

1 P. J. Twin et. al., Phys. Rev. Lett. 57, 811 (1986).

2 S. Chandrasekhar, Ellipsoidal Figures of Equilibrium (Yale University Press, New Haven, 1969) Ch. 7.

3 I. Hamamoto, Phys. Lett. B 143, 31 (1984).

4 H. Frisk and R. Bengtsson, Phys. Lett. B 196, 14 (1987).

5 S. Frauendorf, "Cranking About a Tilted Axis", preprint of invited talk at the Workshop Symposium, Strasbourg, France, March 1991.

6 E. R. Marshalek, Phys. Rev. C 3, 1710 (1971); E. R. Marshalek and M. Sabato, Phys. Rev. C 4, 1006 (1971); E. R. Marshalek and M. Sabato, Phys. Rev. C 5, 1130 (1972).

7 F. Cuypers, Nucl. Phys. A 468, 237 (1987).

8 A. Arima and F. Iachello, The Interacting Boson Model (Cambridge University Press, Cambridge, 1987).

9 E. R. Marshalek and J. Weneser, Phys. Rev. C 2, 1682 (1970); E. R. Marshalek, Nucl. Phys. A275, 416 (1977); E. R. Marshalek, Phys. Rev. 35, 1900 (1987); ibid. 36, 2538 (1987).

${ }^{10}$ A. Klein and E. R. Marshalek, Rev. Mod. Phys. 63, 375 (1991).

${ }^{11} \mathrm{H}$. Ui, Annals of Phys. (NY) 49, 69 (1968).

${ }^{12} \mathrm{R}$. Casten, in Interacting Bose-Fermi Systems in Nuclei, ed. F. Iachello (Plenum, 1981).

${ }^{13}$ T. Mizusaki, N. Yoshinaga and T. Shigahara, Phys. Lett. B 269, 6 (1991).

${ }^{14}$ G. D. Birkhoff, Dynamical Systems (American Mathematical Society, New York, 1966) Vol. IX; G. F. Gustavson, Astron J. 71, 670 (1966). 

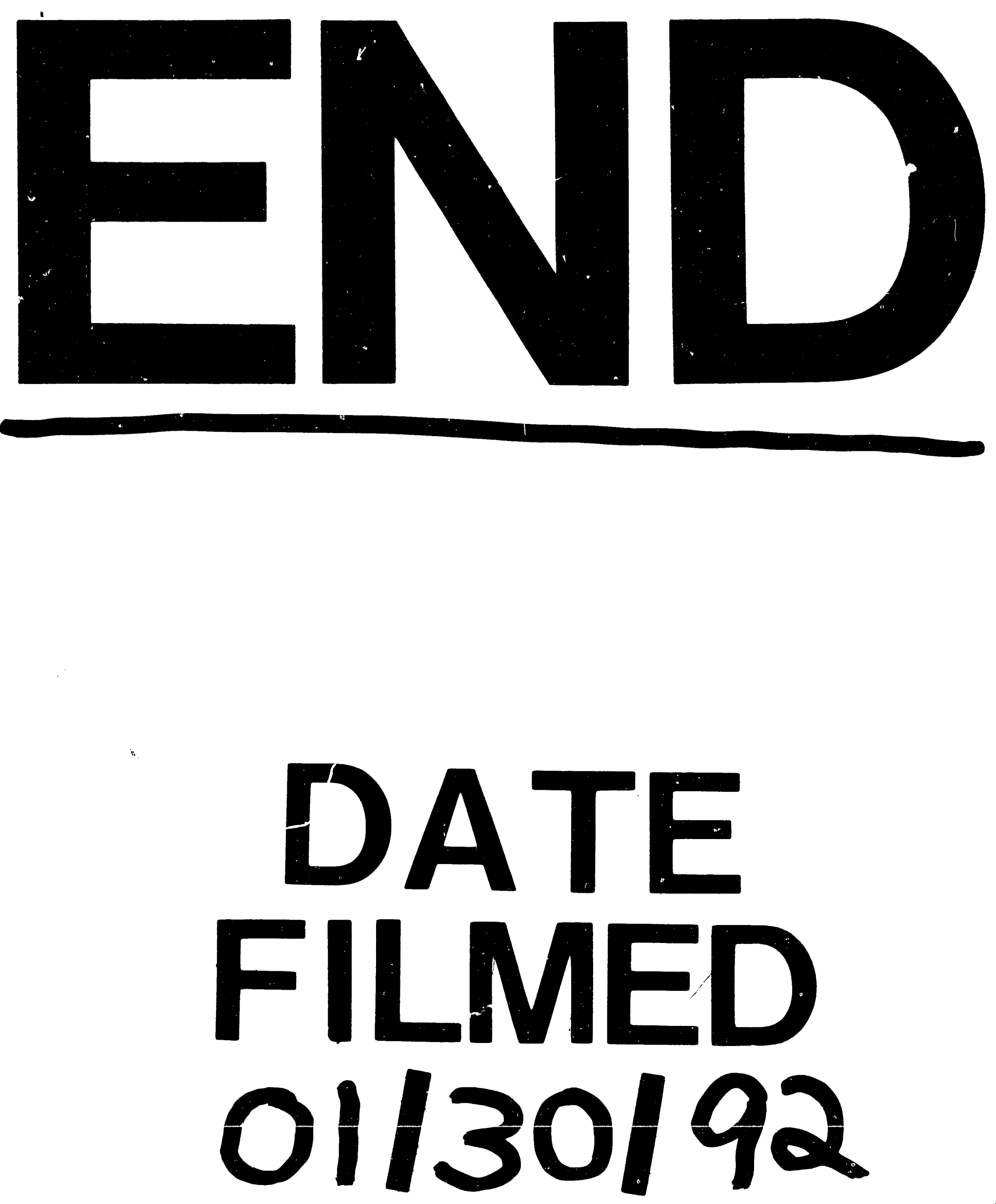


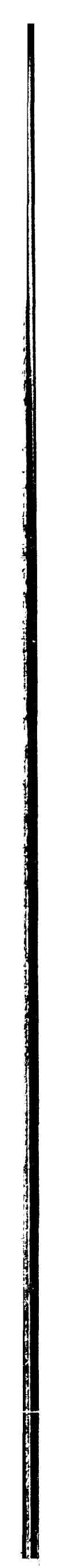

